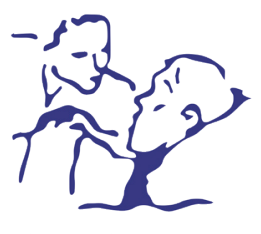

Medicina Paliativa

www.medicinapaliativa.es

ORIGINAL

\title{
Caracterización del dolor en neoplasias hematológicas avanzadas del adulto en la red pública. Ministerio de Salud de Chile
}

\author{
María Lea Derio Palacios', María Elena Cabrera Contreras², Gabriel Cavada Chacón ${ }^{3}$ \\ y Óscar Arteaga Herrera ${ }^{3}$
}

'Departamento de Manejo Integral del Cáncer y otros Tumores, División Prevención y Control de Enfermedades, Subsecretaría de Salud Pública, Ministerio de Salud. ${ }^{2}$ Facultad Medicina, Universidad de Chile, Hospital del Salvador, Santiago. ${ }^{3}$ Escuela Salud Pública, Universidad de Chile.

Recibido el 26 de abril de 2017

Aceptado el 3 de octubre de 2017

\section{PALABRAS CLAVE \\ Cáncer hematológico avanzado, dolor, \\ cuidado paliativo}

\begin{abstract}
Resumen
Introducción: El objetivo del presente trabajo es caracterizar el dolor en neoplasias hematológicas avanzadas del adulto atendidos en la red pública de salud de Chile.

Material y método: Revisión retrospectiva de la base de datos de adultos con neoplasias hematológicas avanzadas el año 2011, que incluye diagnóstico de linfoma, leucemia aguda y crónica, y mieloma que cumplieron los criterios de inclusión, entre ellos intensidad del dolor, a través de la escala visual análoga (EVA) al ingreso y previo al fallecimiento y tipo de dolor. La escala ECOG Performance Status (PS) y Edmonton Symptom Assessment Scale (ESAS) para resto síntomas.

Resultados: De un total de 24.094 pacientes, 771 pacientes (3,2 \%) eran portadores de neoplasia hematológica avanzada, de los cuales 400/771 (52 \%) cumplieron los criterios de inclusión. Las neoplasias hematológicas más frecuentes fueron leucemia y mieloma. Al ingreso, la prevalencia global de dolor fue $88,3 \%$, en mieloma múltiple $96,7 \%$ (134/140 casos), leucemia $87,5 \%$ $(126 / 144)$, linfoma no Hodgkin $80,4 \%(86 / 107)$ y linfoma de Hodgkin 77,8 \% (7/9). El tipo de dolor se asoció al diagnóstico $(p<0,05)$. El tipo de dolor más frecuente fue mixto $56 \%$, solo somático $14 \%$, solo neuropático $13 \%$ y solo visceral 5,3\%. Un 11,7 \% no presentó dolor. Según ECOG al ingreso, el 33 \% era autovalente y el $7 \%$ postrado. La fatiga fue el síntoma más prevalente fuera del dolor. La permanencia media fue de $112 \pm 70$ días, (rango 1-336). El EVA bajó de 6 a 2 puntos $(p<0,05)$ previo al fallecimiento. En el 96,2\% el EVA final, fue menor o igual a $4(p<0,05)$.
\end{abstract}

*Autor para correspondencia:

María Lea Derio Palacios

Dpto. Manejo Integral del Cáncer y otros tumores. División Prevención y Control de Enfermedades. Subsecretaría de Salud Pública. Ministerio de Salud. Mac Iver 541, Santiago, Chile.

Correo electrónico: cancer@minsal.cl 


\section{KEYWORDS}

Advanced

hematological cancer, pain, palliative care.
Conclusiones: La prevalencia de dolor en neoplasia hematológica avanzada fue $88,3 \%$, siendo de 96,7 \% en mieloma. El tipo de dolor se asoció al diagnóstico, siendo el dolor mixto el más frecuente y la fatiga el síntoma más prevalente fuera del dolor. Se logró alivio eficaz del dolor, bajando el EVA de 6 a 2 puntos previo al deceso, y el 96,2 \% de los pacientes alcanzaron un EVA final $\leq 4$.

\section{Abstract}

Aim: The purpose of this study is to characterize pain in adults with advanced hematological malignancies at the public health network of Pain Relief and Palliative Care Program in Chile.

Method: Retrospective review of the database of active patients attended during 2011. Patients with lymphoma, acute and chronic leukemia and multiple myeloma who met the inclusion criteria were included, including pain intensity through visual analogue scale (VAS) at entry and death and type of pain. ECOG Performance Status Scale (PS) and Edmonton Symptom Assessment Scale (ESAS) for rest symptoms.

Results: Out of 24.094 active patients, 771 (3,2\%), had advanced hematological malignancy and $400(52,0 \%)$ met the inclusion criteria. The most common hematologic neoplasia were leukaemia and multiple myeloma. At admission pain was present in $88,3 \%$ of cases, in multiple myeloma $96,7 \%$ (134/140 cases), leukemia 87,5 \% (126/144), non Hodgkin lymphoma 80,4 \% (86/107) and Hodgkin lymphoma 77,8 \% (7/9). Pain type was associated with diagnosis $(p<0,05)$. The most common type of pain was mixed $56 \%$, just somatic $14 \%$, just neuropathic $13 \%$ and just visceral 5,5\%. And 11,7 \% had no pain. According to ECOG at entrance, $33 \%$ were autonomous and $7 \%$ bedridden. Fatigue was the most prevalent symptom besides pain. Mean stay was 112 \pm 70 days (range 1-336). VAS felt from 6 to 2 points $(p<0,05)$ at death. In $96,2 \%$ VAS was equal or less than $4(p<0,05)$ at end of life.

Conclusions: Pain was present in $88,3 \%$ of advanced hematological malignancy, $96,7 \%$ in multiple myeloma. Pain type was associated with diagnosis. Mixed pain was the most common and fatigue the most relevant symptom besides pain. Effective pain relief was achieved, lowering EVA from 6 to 2 points prior to death and $96.2 \%$ of patients achieving a final EVA $\leq 4$.

Derio Palacios ML, Cabrera Contreras ME, Cavada Chacón G, Arteaga Herrera O. Caracterización del dolor en neoplasias hematológicas avanzadas del adulto en la red pública. Ministerio de Salud de Chile. Med Paliat. 2019;26(1):36-42.

\section{Introducción}

Los tumores malignos son la principal causa de mortalidad en el mundo ${ }^{1}$, América $^{2}$ y Chile $^{3}$. Se espera que la incidencia como la mortalidad por cáncer aumente en 2020.

El dolor es uno de los síntomas más temidos en cáncer y está presente en todos los estadios en un alto porcentaje de pacientes: $64 \%$ en enfermedad avanzada/terminal, $59 \%$ en pacientes en tratamiento y $33 \%$ en aquellos curados de cáncer ${ }^{4,5}$.

Respecto a cánceres hematológicos, hay escasa información sobre dolor y cuidados paliativos (CP) en comparación con pacientes portadores de tumores sólidos. Generalmente se sostiene que los pacientes con cánceres hematológicos (leucemia, linfoma y mieloma) presentan dolor en una baja proporción $^{6}$, y se ha reportado que estos son referidos con menor frecuencia y más tardíamente a unidades de CP. Por ejemplo, el grupo de Anderson ${ }^{7,8}$ ha demostrado que el tiempo transcurrido desde el momento de referencia a CP y la muerte fue de 14 días en pacientes hematológicos, comparado con 2-3 meses en aquellos con tumores sólidos. Manitta9 revisó la literatura y confirmó el menor acceso a servicios de $\mathrm{CP}$ de pacientes hematológicos (18\%) en comparación con pacientes con tumores sólidos (44\%).

En la literatura no hay criterios establecidos para catalogar una neoplasia hematológica como "avanzada o refractaria"10; las neoplasias hematológicas tienen características especiales, tales como que la evolución puede variar entre un rápido deterioro clínico y la mejoría, que el equipo tratante tenga metas de curación o expectativas de sobrevida elevadas, que exista un alto compromiso en mantener la intención curativa hasta el final de la vida, la naturaleza potencialmente reversible de eventos intercurrentes como infecciones graves, y también la estrecha relación entre paciente y médico hematólogo ${ }^{9-12}$. Estas razones pueden explicar, en parte, por qué los hematólogos son más reticentes a enviar a sus pacientes a unidades especializadas de cuidados paliativos. El estudio de Hui y cols. ${ }^{13}$ demostró que los hematólogos, con mayor frecuencia que los oncólogos, prescribían terapias de moderada toxicidad, sin beneficio en sobrevida, a pacientes con ECOG 4 y esperanza de vida menor de 1 mes, y sentían una mayor sensación de fracaso 
frente a la enfermedad progresiva en comparación con los especialistas de tumores sólidos.

Sin embargo, Kripp ${ }^{14}$ ha definido tres grupos de riesgo de larga, intermedia o corta sobrevida en neoplasias hematológicas, con la presencia o ausencia de los siguientes factores: necesidad de opioides, mal PS, bajo nivel de albúmina, bajo recuento de plaquetas y deshidrogenasa láctica (LDH) elevada. Bajo riesgo (0-1 factores) sobrevida media 440 días; riesgo intermedio (2-3 factores) sobrevida 63 días y alto riesgo (concurrencia de 4-5 factores) solo 10 días $(p<0,05)$. Odejide $^{15}$, en cambio, plantea que el deterioro de la calidad de vida es el factor más importante para decidir que el paciente ha llegado al final de la vida.

Estos motivos pueden ser la razón de las escasas publicaciones sobre cuidados paliativos y dolor en cáncer hematológico. Los síntomas en estos pacientes están poco documentados. El estudio de $\mathrm{Fadul}^{8}$, comparando 250 pacientes con neoplasias hematológicas y tumores sólidos en su primera consulta a CP, informó que la intensidad del dolor y somnolencia fue similar en ambos grupos, pero hubo mayor incidencia de delirio que en pacientes con tumores sólidos (41 vs. $16 \%$ ). Otros estudios han demostrado que la fatiga es un síntoma frecuente ${ }^{16,17}$ en uno de los cuales constituyó el síntoma más común, $69 \%$. En este estudio, el dolor se observó en un $39 \%$, seguido de síntomas psicológicos como nerviosismo, irritabilidad o tristeza en un $30 \%$. Por su parte, Niscola y cols. ${ }^{18}$, en un grupo de 469 pacientes con neoplasias hematológicas avanzadas tratados en su domicilio, el 52 \% manifestaron dolor, siendo el dolor somático el más prevalente.

La atención de los pacientes con cáncer avanzado en Chile se realiza desde el año $1995^{19,20}$, publicado como Norma Programa Nacional Alivio del Dolor y Cuidados Paliativos (PAD y CP) del Ministerio de Salud (Minsal). El programa se incorporó al Régimen de Garantías Explícitas de Salud (GES) desde el año 2005, que significa el otorgamiento de garantías de acceso, oportunidad y cobertura financiera para todos los pacientes con cáncer avanzado del sistema de salud público y privado de Chile. Este programa provee atención integral y continua, con equipos multidisciplinarios capacitados, quienes evalúan y otorgan tratamiento farmacológico y no farmacológico, junto con información, educación y cuidados al paciente y su familia, desde el momento de su ingreso hasta su fallecimiento.

El objetivo del presente estudio fue caracterizar el dolor en adultos con cáncer hematológico avanzado, que fueron tratados en el PAD y CP del sistema público de salud de Chile, desde el momento del ingreso y previo al fallecimiento, atendidos en el periodo de un año.

\section{Método}

Se realizó una revisión de la base de datos de adultos con cáncer hematológico avanzado, atendidos en las unidades de CP de los 29 servicios de la red pública de salud de Chile. Los antecedentes contenidos en esta base de datos corresponden a la valoración habitual y regular de todos los pacientes, realizada por los equipos de cuidados paliativos. Los datos son extraídos desde la historia clínica en papel o electrónica, e ingresados a la base prospectivamente por los profesionales de estas unidades. Se estudió a los pacientes ingresados a CP, entre el 1 de enero y 3131 de diciembre de 2011. La población atendida por la red pública cubre el $77 \%$ de la población del país.

Los criterios de inclusión fueron: adulto (> de 15 años de edad), atendido en el periodo de estudio, en uno de los 29 servicios de salud, con diagnóstico de cáncer hematológico avanzado, previamente tratados con los protocolos nacionales respectivos (linfoma, leucemia aguda y crónica o mieloma) y derivados al Programa AD y CP por hematólogo tratante. Desde la base de datos se extrae los datos demográficos (edad, sexo) para la evaluación del dolor, los datos de la escala visual análoga (EVA), y para la evaluación del resto de los síntomas, los datos de la Edmonton Symptom Assessment Scale (ESAS). El tipo de dolor se clasificó en nociceptivo (somático y visceral), neuropático y mixto (los dos anteriores juntos) ${ }^{18,21}$. Para evaluar el estado funcional se utilizó la escala del Eastern Cooperative Oncology Group (ECOG) performance status 0-422. El dolor, otros síntomas y ECOG fueron evaluados en la fecha de ingreso al programa por el médico y la enfermera; y en la última semana de vida, se evaluó el dolor con EVA por médico, enfermera o familiar cuidador capacitado. Se excluyeron los casos que carecían de los datos mencionados anteriormente y aquellos que al cierre del estudio no habían fallecido.

La terapia analgésica utilizada incluyó desde analgésicos antinflamatorios no esteroidales (AINE) hasta opioides débiles y opioides fuertes, según EVA del paciente y en base a los Protocolos y Guía Clínica del PAD y CP del Minsal23. Estos se basan en las guías de la escala analgésica de la OMS que continúa siendo el gold standard para el alivio del dolor por cáncer y las recomendaciones de la Asociación Europea de Cuidados paliativos ${ }^{24,25}$.

\section{Aspectos éticos}

La base de datos de cada centro PAD y CP de la red pública contiene los antecedentes de todos los pacientes ingresados al programa, corresponden a la valoración habitual y regular de todos los pacientes, realizada por los equipos de cuidados paliativos, los datos son extraídos desde la historia clínica en papel o electrónica, e ingresados a la base prospectivamente por los profesionales de estas unidades. Se envía mensualmente al Ministerio de Salud (MINSAL), es de uso restringido y conserva el anonimato de las personas. Todos los pacientes, o su cuidador, firmaron consentimiento informado $(\mathrm{Cl})$ para ingresar al Programa.

\section{Análisis estadístico}

Las variables analizadas fueron: diagnóstico hematológico, edad, sexo, EVA, ESAS, ECOG y tipo de dolor al ingreso y EVA la semana previa al deceso. Las variables numéricas se describieron mediante percentiles, promedio y desviación estándar, y comparadas mediante análisis de la varianza (ANOVA) con comparaciones múltiples basadas en el test de Bonferroni; las variables nominales fueron descritas mediante frecuencias y comparadas con el test de independencia de Chi cuadrado; y las variables ordinales 
mediante percentiles y comparadas mediante el test de Kruskall-Wallis. El cambio de EVA entre ingreso y deceso fue evaluado a través del test de Wilcoxon y se utilizó la correlación de Spearman para asociar variables ordinales y estas con variables numéricas. Se utilizó nivel de significación de $5 \%$. Los datos fueron procesados en el programa estadístico STATA versión 13.0.

\section{Resultados}

Durante el año 2011, se atendieron en el PAD y CP 24.094 pacientes (adultos y niños), de ellos 771 (3,2 \%) eran portadores de cáncer hematológico avanzado. El resto, 23.323 casos $(96,8 \%)$, correspondía a tumores sólidos. De los 771 casos, $400(51,9 \%)$ eran adultos y cumplían los requisitos de inclusión. El resto se excluyó por las siguientes razones: no habían fallecido $25,9 \%(200 / 771)$, ausencia de registro EVA de ingreso $15,7 \%(121 / 771)$ y edad menor a 15 años 6,5 \% (50/771). El diagnóstico de los casos excluidos fue similar al de los analizados.

En la distribución por sexo, se observó un leve predominio de mujeres, 51,5 \% (206 casos). La edad promedio de los varones fue $67,6 \pm 12$ años y de las mujeres $63,0 \pm 14$, $(p<0,05)$. La distribución de los diagnósticos fue la siguiente: leucemia aguda y crónica ( $L ; n=144,36,0 \%$ ), mieloma múltiple (MM; $n=140,35 \%)$, linfoma no Hodgkin $(\mathrm{LNH} ; \mathrm{n}=107,27,0 \%)$ y linfoma de Hodgkin (LH; $n=9,2,0 \%)$. La distribución según diagnóstico se presenta en la Figura 1. Los pacientes con diagnóstico de MM y LNH fueron de mayor edad (70 años), respecto a aquellos con L (62 años) y a su vez de LH (47 años). Respecto al ECOG al ingreso, el $33 \%$ (132 casos) estaba en PS 0 , el 15,0\% (60 casos) en PS 1 , el $15,0 \%$ (60 casos) en PS 2 , el 30,0 \% (120 casos) en PS 3 y el $7,0 \%$ (28 casos) en PS 4 . No hubo registro de pacientes con diagnóstico de otras neoplasias hematológicas como síndro- me mielodisplástico, trombocitemia esencial o policitemia vera y tampoco rechazos.

La distribución de los síntomas al ingreso (excepto el dolor), según diagnóstico, se observa en la Figura 2. Los síntomas más frecuentes, fuera del dolor, fueron fatiga, baja de peso, insomnio, anorexia y tristeza. Además de estos, la disnea y hemorragia fueron síntomas frecuentes de leucemia. La concomitancia de síntomas fue cuatro por paciente en leucemia y tres en MM y linfoma.

La prevalencia de dolor fue del 88,3\% al ingreso del Programa. Las patologías que presentaron mayor dolor en orden decreciente fueron: MM 96,7 \% (134/140 casos), L $87,5 \%$ (126/144), LNH 80,4 \% (86/107) y LH 77,8 \% (7/9). El tipo de dolor se asoció significativamente al diagnóstico $(p<0,05)$. En la Tabla I se muestra el tipo de dolor de acuerdo al diagnóstico. El tipo de dolor más frecuente en todas las neoplasias fue el dolor mixto 56 \% (224/400). Sin embargo, en $M M$ fue prevalente el dolor neuropático exclusivo $(30,7 \%)$, en cambio en linfoma y leucemia fue el somático (11-20\%). Cabe hacer notar que 11,8 \% (47 casos) no presentó dolor, principalmente en ambos subtipos de linfoma.

La intensidad del dolor, evaluada como EVA al ingreso y previo al deceso, bajó en forma significativa, con una mediana inicial de 6 puntos (dolor moderado) y final de 2 (dolor leve), $(p<0,05)$. Previo al momento del deceso, el 88,5\% declaró un EVA máximo de 3 puntos (dolor leve) y el 96,3 $\%$ un EVA máximo de 4 puntos (Tabla II). No se observó una asociación significativa entre la intensidad del dolor y el PS, tampoco entre PS y edad. Las medianas de PS fueron iguales a 2 en ambos sexos. No se evidenció diferencias del EVA final por tipo de tumor.

Los pacientes permanecieron en el Programa en promedio $112 \pm 70$ días (rango 1-336). Los pacientes con MM $143 \pm 73$ días, LNH $107 \pm 68$ días, LH $104 \pm$ 33,4 días y L $93 \pm 63$ días, sin diferencia significativa entre ellos.

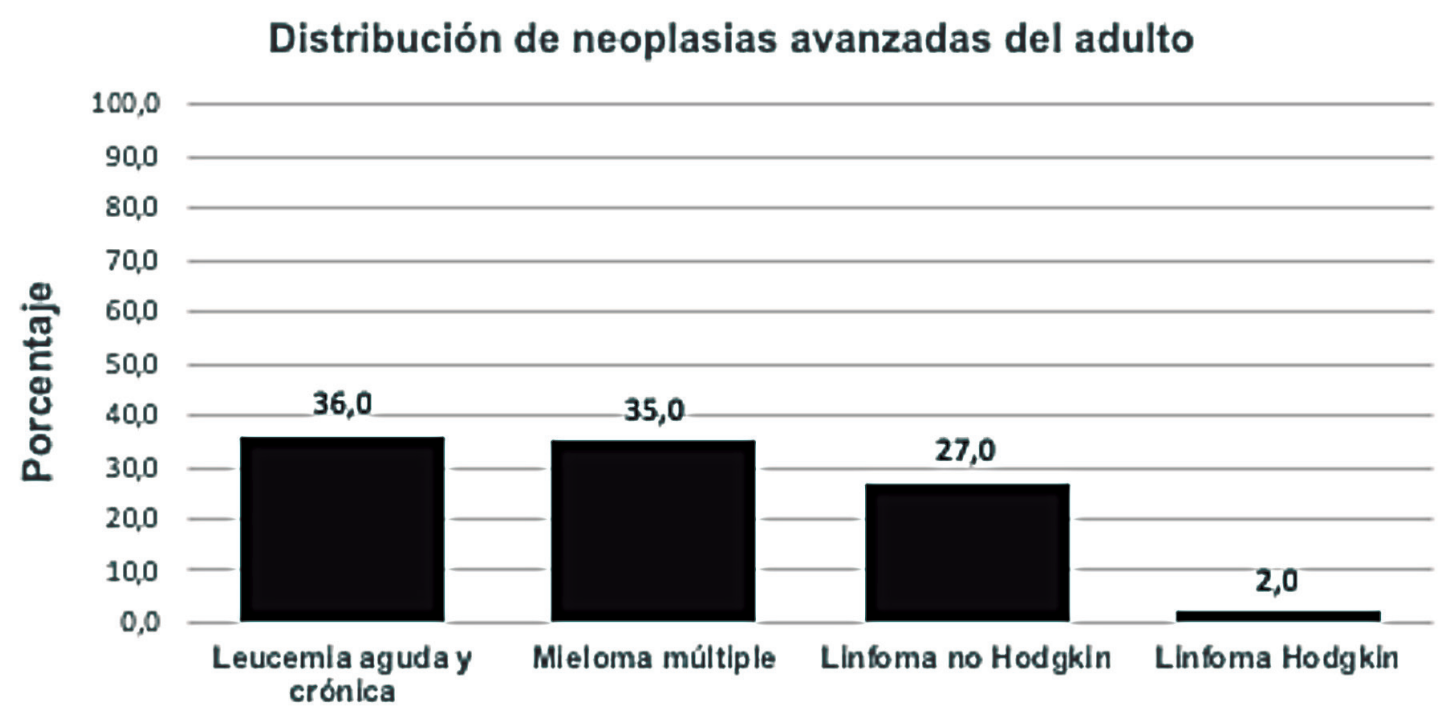

Figura 1. Distribución de neoplasias hematológicas avanzadas del adulto. 


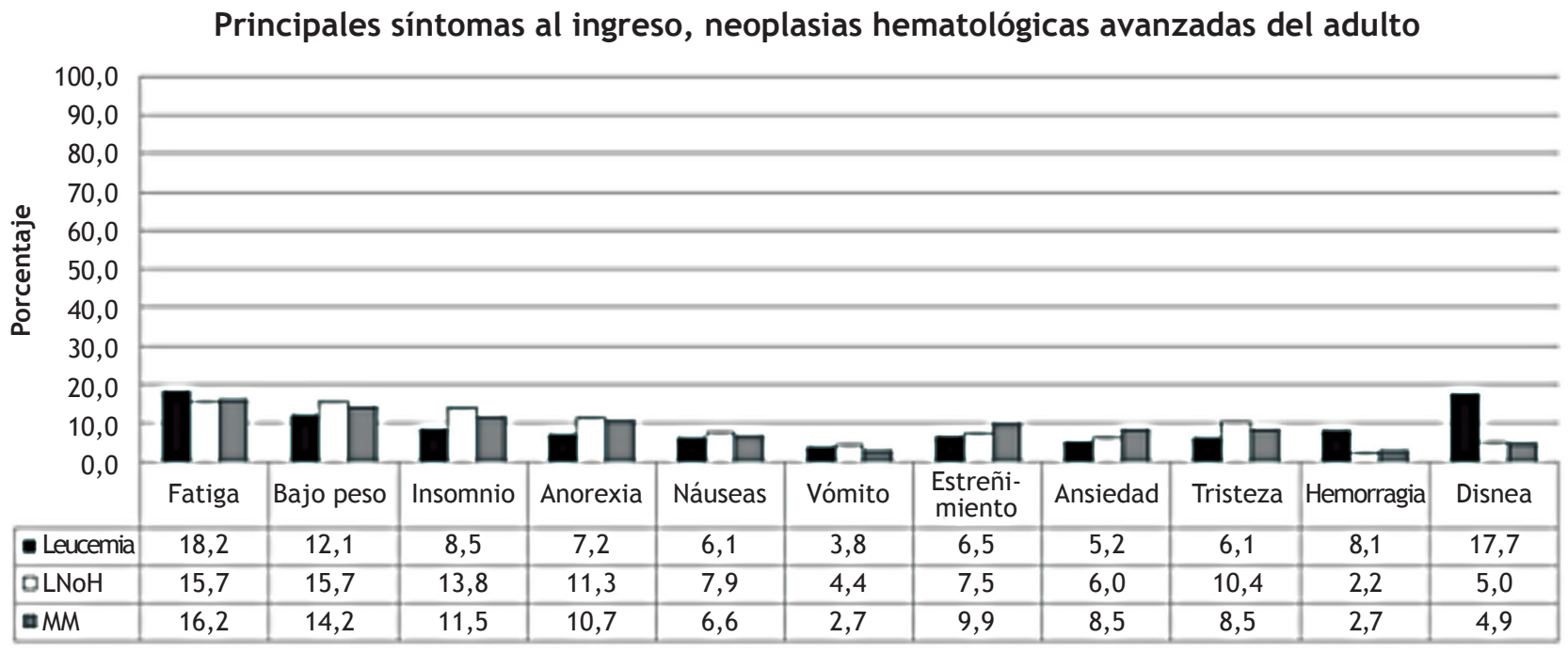

Figura 2. Principales síntomas según neoplasia hematológica avanzada del adulto.

Tabla I. Tipo de dolor al ingreso según neoplasia hematológica avanzada del adulto.

\begin{tabular}{|c|c|c|c|c|c|c|}
\hline \multirow{2}{*}{\multicolumn{2}{|c|}{$\begin{array}{c}\text { Tipo de dolor } \\
\text { N (\%) }\end{array}$}} & Leucemia & $\begin{array}{l}\text { Linfoma } \\
\text { Hodgkin }\end{array}$ & $\begin{array}{c}\text { Linfoma no } \\
\text { Hodgkin }\end{array}$ & $\begin{array}{l}\text { Mieloma } \\
\text { Múltiple }\end{array}$ & Total \\
\hline & & N (\%) & $\mathrm{N}(\%)$ & $\mathrm{N}(\%)$ & $\mathrm{N}(\%)$ & \\
\hline \multirow{6}{*}{ 응 } & Mixto & $87(60,4)$ & $4(44,4)$ & $54(50,5)$ & $79(56,4)$ & $224(56,0)$ \\
\hline & Somático & $29(20,1)$ & $1(11,1)$ & $15(14,0)$ & $11(7,9)$ & $56(14,0)$ \\
\hline & Visceral & $6(4,2)$ & $1(11,1)$ & $13(12,2)$ & $1(0,7)$ & $21(5,3)$ \\
\hline & Solo neuropático & $4(2,8)$ & $1(11,1)$ & $4(3,7)$ & $43(30,7)$ & $52(13,0)$ \\
\hline & Sin dolor & $18(12,5)$ & $2(22,2)$ & $21(19,6)$ & $6(4,3)$ & $47(11,8)$ \\
\hline & Total & $144(100,0)$ & $9(100,0)$ & $107(100,0)$ & $140(100,0)$ & $400(100,0)$ \\
\hline
\end{tabular}

\section{Discusión}

Los pacientes con neoplasias hematológicas avanzadas constituyeron solo el 3,2 \% de los casos que fueron referidos al Programa AD y CP, del Ministerio de Salud de Chile, es decir, menos de la mitad de su incidencia si consideramos que estas neoplasias constituyen aproximadamente el $9 \%$ de todos los tumores ${ }^{26}$. Esta situación está en concordancia con los datos de la literatura, en los cuales los pacientes con neoplasias hematológicas tienen menor acceso a las unidades de CP y, por tanto, no se benefician de la atención de un equipo multidisciplinario en la etapa final de la vida. En un estudio en Alemania ${ }^{27}$, las neoplasias hematológicas, constituyeron solo el $5 \%$ de las referencias. En cambio en Australia ${ }^{28}$ el número de referencias de casos hematológicos aumentó de 5,3\% a 11,6 \%, en un periodo de 4 años, 2007-2010, debido a una mayor colaboración entre Hematología y CP, con resultados potencialmente positivos para los pacientes.

El dolor se observó en 88,3\% de los pacientes en nuestra serie, llegando al 96,7 \% en MM. En forma similar, los estudios de Bandieri y cols. ${ }^{11}$ y Niscola y cols. ${ }^{18}$ demuestran que el MM es la patología hematológica con mayor incidencia de dolor, 90 y $86 \%$, respectivamente, aunque hay trabajos que reportan menor incidencia, 43-73 \%29-31. El dolor se debe a destrucción ósea por aumento de la actividad osteoclástica y ausencia de actividad osteoblástica, produciendo fracturas patológicas y compresión medular ${ }^{18,21}$. En nuestro estudio el dolor fue exclusivamente neuropático en $31 \%$ de los pacien- 
Tabla II. EVA ingreso y final en neoplasia hematológica avanzada del adulto

\begin{tabular}{ccc}
\hline Valor EVA & $\begin{array}{c}\text { Pacientes } \\
\text { evaluados al } \\
\text { ingreso }\end{array}$ & $\begin{array}{c}\text { Pacientes } \\
\text { evaluados al } \\
\text { egreso }\end{array}$ \\
\cline { 2 - 3 } $\mathbf{N}(\%)$ & $\mathbf{N}(\%)$ \\
\hline 1 & $47(11,8)$ & $94(23,5)$ \\
2 & $18(4,5)$ & $46(11,5)$ \\
3 & $34(8,5)$ & $93(23,3)$ \\
4 & $34(8,5)$ & $121(30,3)$ \\
5 & $48(12,0)$ & $31(7,8)$ \\
6 & $50(12,5)$ & $12(3,0)$ \\
7 & $46(11,5)$ & $3(0,8)$ \\
8 & $59(14,8)$ & $0(0,0)$ \\
9 & $60(15,0)$ & $0(0,0)$ \\
10 & $2(0,5)$ & $0(0,0)$ \\
& $2(0,5)$ & $0(0,0)$ \\
\hline $\mathrm{p}<0,05$, para la diferencia. & 400 \\
\hline
\end{tabular}

tes con MM, es decir, por compresión de estructuras nerviosas principalmente de la columna vertebral. Los pacientes con mieloma son referidos a CP en forma más precoz en Chile, frecuentemente en forma concomitante con el inicio de la quimioterapia, hecho que se ve reflejado en el mayor tiempo de permanencia en el Programa, en comparación con las otras patologías.

Respecto a leucemia y linfoma, nuestro estudio reveló una alta frecuencia de dolor, desde $87,5 \%$ en leucemia, $80,4 \%$ en LNH y $77,8 \%$ en Hodgkin, al igual que en la serie de Bandieri y cols., que describe la presencia de dolor en $83 \%$ de casos con leucemia y linfoma. Este hallazgo confirma que el dolor es un síntoma prevalente en estas neoplasias hematológicas, excluido el mieloma, y descarta la creencia que el dolor es infrecuente en el cáncer hematológico ${ }^{20}$.

Un hallazgo interesante fue observar que $12 \%$ de todos los pacientes no tiene dolor al inicio, en especial en linfoma, en el que la cifra llega al $20 \%$. La idea general de que las neoplasias hematológicas sufren poco dolor, se refiere probablemente a las neoplasias linfoproliferativas, como lo confirma este trabajo.

Los demás síntomas que presentaron estos pacientes fueron similares a los descritos en tumores sólidos. Por ejemplo, entre los más frecuentes fueron fatiga y baja de peso, al igual que los estudios de Le Blanc y cols. ${ }^{16}$ y Manitta y cols. ${ }^{17}$, llegando casi al $70 \%$ en este último, y por lo tanto la misma necesidad de cuidado. La etiología de la fatiga es multifactorial y poco comprendida, con varios estudios que muestran una fuerte correlación entre fatiga, síntomas psicológicos y dolor. Entre las causas de la fatiga están la anemia, pero también factores sicológicos como ansiedad y depresión ${ }^{30,31}$.
La prevalencia de síntomas psicológicos fue relativamente baja en nuestro estudio, ansiedad (5-8\%) y tristeza (6-10\%), comparado con otros estudios en los cuales llega al $30 \%{ }^{16}$, probablemente por falta de registro dirigido. El delirio y la somnolencia se reportaron, además, en otro estudio ${ }^{8}$, más frecuente que en casos con tumores sólidos. La disnea y sangrado fueron síntomas observados con mayor prevalencia en leucemia en nuestra casuística.

En el presente trabajo se muestra una disminución del dolor, desde moderado $(E V A=6)$ al ingreso a leve (EVA = 2) en la semana previa al fallecimiento. El 88,5\% de los pacientes manifestaron un EVA menor a 3 al final de la vida. Nuestro estudio es comparable con el realizado en Italia en 469 pacientes con neoplasias hematológicas avanzadas ${ }^{17}$, que logró control de los síndromes dolorosos en $92 \%$.

En suma, este estudio reafirma que el dolor es un síntoma prevalente en las neoplasias hematológicas, principalmente en mieloma múltiple; que la intervención sobre el dolor es eficaz logrando reducir significativamente el dolor, entre el ingreso y los días previos al fallecimiento del paciente; que es necesaria la capacitación y unificación de criterios entre los profesionales respecto de los datos a consignar en la ficha clínica, aunque esta sea estructurada; que es indispensable mayor colaboración y coordinación entre los hematólogos y las Unidades de CP, para una atención integrada de estos pacientes, siendo perfectamente posible la coexistencia de tratamientos curativos con tratamientos sintomáticos/ paliativos.

\section{Limitaciones}

Este estudio describe algunas características del síntoma dolor en un grupo de pacientes que comparten una condición (neoplasias hematológicas avanzadas), en un momento específico del tiempo (entre el 1 de enero y el 31 de diciembre de 2011), pero no pueden establecer asociaciones de causalidad (causa-efecto). La gran limitación de este tipo de estudio es la ausencia de un grupo control.

Esta evaluación considera solo a los pacientes de la red pública y omite al sistema privado. Sin embargo, en Chile no hay razones para pensar que las personas atendidas en el sistema privado vayan a tener un mejor resultado.

\section{Financiación}

Ministerio de Salud, Departamento de Cáncer. Conflictos de intereses

No existen conflictos de intereses.

\section{Agradecimientos}

Dra. Marisol Ahumada Olea, Anestesióloga, Asesora Programa Nacional Alivio del Dolor por cáncer y Cuidados Paliativos del Ministerio de Salud de Chile, por sus comentarios sobre el manuscrito. 


\section{Bibliografía}

1. World Health Organization. Globocan. International Agency for Reseach on Cancer; 2012.

2. Organización Panamericana de la Salud. Situación de Salud en las Américas 2012. Disponible http: / / www.paho.org.

3. Proyecciones de Mortalidad en Chile 2011 al 2020, para algunas causas no transmisibles en vigilancia del Departamento de Epidemiología. Ministerio de Salud; 2013.

4. Van den Beuken-van Everdingen MH, de Rijke JM, Kessels AG, Schouten HC, Van Kleef M, Patijn J. Prevalence of pain in patients with cancer: a systematic review of the past 40 years. Ann Oncol. 2007;18(9):1437-49.

5. Reyes D, González JC, Mohar A, Meneses A. Epidemiología del dolor por cáncer. Rev. Soc. Esp. Dolor. 2011;18(2):118-34.

6. Serrano-Bermudez G, Porta-Sales J, González-Barboteo J, Garzón-Rodríguez C, Pallejà Pejoan M, Fernández de Sevilla A. Neoplasias hematológicas y cuidados paliativos: revisión sistemática de la literatura. Med Paliat. 2012;19(2):73-80.

7. Cheng WW, Willey J, Palmer JL, Zhang T, Bruera E. Interval between palliative care referral and death among patients treated at a comprehensive cancer center. J Palliat Med. 2005;8(5):1025-32.

8. Fadul NA, El Osta B, Dalal S, Poulter VA, Bruera E. Comparison of symptom burden among patients referred to palliative care with hematologic malignancies versus those with solid tumors. J Palliat Med. 2008;11(3):422-7.

9. Manitta VJ, Philip JAM, Cole-Sinclair MF. Palliative Care and the Hemato-Oncological Patient: Can We Live Together? A Review of the Literature. J Palliat Med. 2010;13(8):1021-5.

10. Glare P. A systematic review of physicians' survival predictions in terminally ill cancer patients. BMJ. 2003;327(7408):195-8.

11. Bandieri E, Sichetti D, Luppi M, Di Biagio K, Ripamonti C, Tognoni $G$, et al. Is pain in patients with haematological malignancies under-recognised? The results from Italian ECAD-O survey. Leuk Res. 2010;34(12):334-5.

12. Selvaggi KJ, Vick JB, Jessell SA, Lister J, Abrahm JL, Bernacki R. Bridging the gap: a palliative care consultation service in a hematological malignancy-bone marrow transplant unit. J Community Support Oncol. 2014;12(2):50-5.

13. Hui D, Bansal S, Park M, Reddy A, Cortes J, Fossella F, et al. Differences in attitudes and beliefs toward end-of-life care between hematologic and solid tumor oncology specialists. Ann Oncol. 2015;26(7):1440-6.

14. Kripp M, Willer A, Schmidt C, Pilz LR, Gencer D, Buchheidt D, et al. Patients with malignant hematological disorders treated on a palliative care unit: prognostic impact of clinical factors. Ann Hematol. 2014;93(2):317-25.

15. Odejide OO, Salas Coronado DY, Watts CD, Wright AA, Abel GA. End-of-Life care for blood cancers: a Series of focus groups with hematologic oncologists. J Oncol Pract. 2014;10(6):e396-e403.

16. LeBlanc TW, Smith JM, Currow DC. Symptom burden of haematological malignancies as death approaches in a community palliative care service: a retrospective cohort study of a consecutive case series. Lancet Haematol. 2015;2(8):334-8.

17. Manitta V, Zordan R, Cole-Sinclair M, Nandurkar H, Jennifer Philip J. The Symptom Burden of Patients with Hematological Malignancy: A Cross-Sectional Observational Study. J Pain Symptom Management. 2011;42(3):432-42.
18. Niscola P, Cartoni C, Romani C, Brunetti GA, D'Elia GM, Cupelli $\mathrm{L}$, et al. Epidemiology, features and outcome of pain in patients with advanced hematological malignancies followed in a home care program: an Italian survey. Ann Hematol. 2007:86(9):671-6.

19. Norma General Técnica Nacional N. ${ }^{\circ} 31$, Norma de Enfermería (Resolución Exenta $\mathrm{N}^{\circ} 1961$ del Depto. Asesoría Jurídica Ministerio de Salud de Chile) y N. ${ }^{\circ} 32$, publicada con el nombre de Norma Nacional Alivio del Dolor y Cuidados Paliativos (Departamento Jurídico del Ministerio de Salud, por Resolución Exenta $N .^{\circ} 129$ ).

20. Decreto N. ${ }^{\circ} 4$, del 5 de febrero del 2013, Aprueba Garantías Explicitas en Salud del Régimen de General de Garantías en Salud de los Ministerios de Salud y Hacienda, AUGE 80 Problema N. ${ }^{\circ} 4$.

21. Aguilar JL, Guanyabens C, Romero P, Peláez R, Fernández S, Mata J, et al. Dolor en hematología clínica. Rev Soc Esp Dolor. 2010;17(1):32-50.

22. Loprinzi CL, Laurie JA, Wieand HS, Krook JE, Novotny PJ, Kugler JW, et al. Prospective evaluation of prognostic variables from patient-completed questionnaires. J Clin Oncol. 1994;12(3):601-7.

23. Ministerio de Salud de Chile: Guía Clínica AUGE, Alivio del dolor por Cáncer y CuidadosPaliativos, disponible:http://web. minsal.cl/portal/url/item/72213ed52c2723d1e0400101 1f011398.pdf.

24. Caraceni A, Cherny N, Fainsinger R, Kaasa S, Poulain P, Radbruch $\mathrm{L}$, et al. Pain Measurement Tools and Methods in Clinical Research in Palliative Care: Recommendations of an Expert Working Group of the European Association of Palliative Care. J Pain Symptom Manage. 2002;23(3):239-55.

25. Caraceni A, Hanks G, Kaasa S, Bennett MI, Brunelli C, Cherny $\mathrm{N}$, et al. Use of opioid analgesics in the treatment of cancer pain: evidence-based recommendations from the EAPC. Lancet Oncol. 2012;13(2):58-68.

26. Globocan 2012: Estimated Cancer Incidence, Mortality and Prevalence Worlwide in 2012. http://globocan.iarc.fr/Pages/ fact_sheets_population.aspx.

27. Corbett CL, Johnstone M, McCracken Trauer J, Spruyt O. Palliative Care and Hematological Malignancies: Increased Referrals at a Comprehensive Cancer Centre. J Palliat Med. 2013;16(5):537-41.

28. Alt-Epping B, Wulf G, Nauck F. Palliative care for patients with hematological malignancies-a case series. Letter to the Editor. Ann Hematol. 2011;90:613-5.

29. Porta-Sales J, Nabal-Vicuña M, Vallano A, Espinosa J, PlanasDomingo J, Verger-Fransoy E, et al. Have We Improved Pain Control in Cancer Patients? A Multicenter Study of Ambulatory and Hospitalized Cancer Patients. J Palliat Med. 2015;18(11):923-32.

30. Kiely F, Cran A, Finnerty D, O'Brien T. Self-Reported Quality of Life and Symptom Burden in Ambulatory Patients With Multiple Myeloma on Disease-Modifying Treatment. Am J Hosp Palliat Care. 2017;34(7):671-6.

31. Romito F, Montanaro R, Corvasce C, Di Bisceglie M, Mattioli V. Is cancer-related fatigue more strongly correlated to haematological or to psychological factors in cancer patients? Support Care Cancer. 2008;16(8):943-6. 